\title{
Determination of Limited Aritolonchic Acid A Content in Xinqin Granules by Capillary Electrophoresis
}

\author{
Fang Liu ${ }^{1,}$, Haixing $\operatorname{Liu}^{2, \mathrm{~b}}$ and Likun $\mathrm{Han}^{2, \mathrm{c}^{*}}$ \\ ${ }^{1}$ Department of Chemical Engineering, Weifang Vocational College, Weifang 262737, China \\ ${ }^{2}$ Chemistry \& Chemical \& Environmental Engineering College, Weifang University, Weifang 261061, China
}

\begin{abstract}
The paper investigated the determination of limited aritolonchic acid A content in Xinqin granules by capillary electrophoresis. The buffer was $30 \mathrm{mmol} / \mathrm{L}$ borax solution containing $3 \%$ ethanol. The voltage was $16 \mathrm{kV}$ and UV detection wavelength was $250 \mathrm{~nm}$. The aritolonchic acid A content in Xinqin granules was $2.87 \mathrm{mg} / \mathrm{g}(\mathrm{RSD}=2.9 \%)(\mathrm{n}=6)$. The recovery of aritolonchic acid A in Xinqin granules sample was in the range of $84.5 \%-126.7 \%(n=5)$. The average recovery was $105.9 \%(n=5)$. The new method is fit for the determination of limited aritolonchic acid A content in Xinqin granules.
\end{abstract}

\section{Introduction}

Xinqin granules are consists of manchurian wildginger, baical skullcap root, fineleaf schizonepeta herb, dahurian angelica root, cassia twig, siberian cocklour fruit, grassleaf sweelflag rhizome, membranous milkvetch root, largehead atractylodes rhizome and divaricate saposhnikovia root. It has tonifying qi and strengthening exterior, dispelling wind and relieving stuffy nose. It is used for treatment of nose itching, sneezing, runny nose and allergic rhinitis by lung qi deficiency and wind- pathogen. Aristolochic acid A has become a hot topic that it maybe has liver and kidney toxicity[1]. Wang Liang et al [2] researched the effects of the dissolution of aristolochic acid A in caulis aristolochiae manshuriensis by different doses of bamboo leaf. The experiments showed that bamb $b^{1}$ oo leaf is obvious for reducing the dissolution of aristolochic acid A. Liu Mei-feng et al [3] investigated extract process of aristolochic debilis and toxicity reduction by different processing using HPLC. They studied four factor effect of Aristolochic debilis, decoction number, amount of water and decoction time. Zhang Ping [4] compared the aristolochic acid A content of leaf, stem and root of Saruma by HPLC. The result will contribute to the utilization of Sarume drug and reduce its toxicity. Qiu Quan et al [5] determined the content of aristolochic acid $\mathrm{A}$ in Shennong snake liquor by HPLC. The Accucore C18

(2.6 $\mu \mathrm{m}, 3 \mathrm{~mm} \times 100 \mathrm{~mm})$ column was used with mobile phase A ( $0.1 \%$ phosphoric acid solution) and mobile phase B (acetonitrile) at the flow of 0.3 $\mathrm{mL} / \mathrm{min}$ by gradient elution. The wavelength was 250 $\mathrm{nm}$ and sampling volume was $10 \mu \mathrm{L}$. Ge Xiaozhong et

${ }^{*}$ Corresponding author mail: chan77116@wfu.edu.cn a27255116@qq.com; bliuhx@wfu.edu.cn al [6] studied the limit determination of aristolochic acid A in Xinqin granules using HPLC. The analysis was performed on the $\mathrm{C} 18$ column $(5 \mu \mathrm{m}, 4.6 \mathrm{~mm} \times 150$ $\mathrm{mm})$ and methanol-acetic acid-water (55:2:43) mobile phase $(1.0 \mathrm{ml} / \mathrm{min}$ flow $)$ at $387 \mathrm{~nm}$ wavelength. Pan Yankun et al [7] explored a method for the determination of aristolochic acid A in several traditional Chinese medicine by LC-MS/MS. The medicine sample solution was obtained by acetone ultrasonically extracting and evaporating dryness heated in a water bath (below $50^{\circ} \mathrm{C}$ ). The sample solvent and mobile phase was the same solution (water: acetic acid: acetonitrile =49:1:50). The Kromasil C18 column was used. It was shown that the content of aristolochic acid A in Xungufeng formula was higher and it was careful for using the medicine. Li Wei et al [8] explored the determining aristolochic acid A content in Rhizoma Asari and Yangxue Qingnao Granules by LC-MS/MS. The Agilent XDB-C18 column $(5 \mu \mathrm{m}, 2.1 \mathrm{~mm} \times 150 \mathrm{~mm})$ was used with mobile phase of methanol-0.1\% formic acid(90:10). Zhou Wei et al [9-10] studied aristolochic acid A component in Chinese patent medicine (Xinyi pill, Xiaoqinglong pill, Bazhengsan pill and Stephania) by HPLC/MS. The COSMOS C18-MS $(5 \mu \mathrm{m}, 4.6 \mathrm{~mm}$ $\times 250 \mathrm{~mm})$ was used with Methanol-water-acetic $\operatorname{acid}(64: 35: 1, \mathrm{v} / \mathrm{v})$ at $0.8 \mathrm{~mL} / \mathrm{min}$ velocity of flow. The detection wavelength was $310 \mathrm{~nm}$. They did not detect aristolochic acid A in Xiaoqinglong pill. In another of their articles, there were not detect aristolochic acid A in Duhuo Jisheng pill, Longdan Xiegan pill, Guipi pill, Xiangsha Yangwei pill, Shujin Huoxue pill, Xianglian pill and Xiangsha Liujun pill. Nan Tiegui et al [11] investigated aristolochic acid A-bovine serum albumin conjugate and aristolochic acid A-ovalbumin conjugate as the immunogen and coating antigen, respectively. A sensitive monoclonal antibody-based 
indirected enzyme linked immunosorbent assay was developed and used for the determination of aristolochic acid $\mathrm{A}$ in six kinds of Chinese herbal medicines and five kinds of Chinese patent medicines (Shuangxiang Paishi granules, Longdan Xiegan pill, Dieda pill, Lianqiao Baidu pill and Zhisou Qingguo pill, but, all no detecting). Lu Qinghua [12] explored the limit detecting of aristolochic acid A in Zhuifeng Tougu capsule by HPLC. The Water C18(5 $\mu \mathrm{m}, 4.6$ $\mathrm{mm} \times 250 \mathrm{~mm}$ ) was used with a mixture of acetonitrile and $0.4 \%$ acetic acid (30:70) as mobile phase. The wavelength was $393 \mathrm{~nm}$. In this article, the aritolonchic acid A content in Xinqin granules was determined by Capillary Electrophoresis.

\section{Experimental section}

\subsection{Instruments and Reagents}

Instruments: CL 1030 capillary electrophoresis instrument (Beijing cailu company); The capillary (75 $\mu \mathrm{m}, 52 / 44 \mathrm{~cm}$ length (overall/effective) from Hebei Yongnian Ruifeng company.

Aritolonchic acid A comes from Chinese Drugs and Biological Products; Xinqin granules (Zhejiang youshengmeite medicine company).

\subsection{Experimental Methods}

At first, the capillary tube is rinsed in sequence using $1 \mathrm{~mol} \cdot \mathrm{L}^{-1}$ hydrochloric acid, purified water, $1 \mathrm{~mol} \cdot \mathrm{L}^{-1}$ sodium hydroxide, purified water and buffer for $5 \mathrm{~min}$ each. After three injections, the capillary was handled using the above method again.

The voltage was chosen $16 \mathrm{kV}$ and UV detection wavelength was $250 \mathrm{~nm}$. Sample injection $(7.5 \mathrm{~cm}$ height) time was $10 \mathrm{~s}$.

\subsection{Sample Preparation}

Xinqin particle sample solution: Accurate Xinqin particle $1.7399 \mathrm{~g}$ was put in $40 \mathrm{~mL}$ methanol - water (1:1) and extracted $24 \mathrm{~h}$ at $18^{\circ} \mathrm{C}$. The sample solution was obtained by filtering and fixing volume $50 \mathrm{~mL}$.

Aritolonchic acid A standard solution: Aritolonchic acid A was accurately weighed $0.0012 \mathrm{~g}$, added $2 \mathrm{~mL}$ methanol and Ultrasonic wave assisted dissolution for 10 minutes.

\section{Results and Discussion}

\subsection{Selection electrophoresis conditions}

The experiment voltage was selected $16 \mathrm{kV}$. The UV wavelength was $250 \mathrm{~nm}$.

The experiment buffer was $30 \mathrm{mmol} / \mathrm{L}$ borax solution (contain 3\% ethanol) by trial.

\subsection{Quantitative analysis}

\subsubsection{Standard curve.}

The aritolonchic acid A standard solution was prepared and the concentrations were $0.6,0.3,0.15$, $0.075,0.0375,0.0188,0.0094 \mathrm{mg} / \mathrm{mL}$. Each standard solution was tested three times. The aritolonchic acid A chromatogram was showed in Figure 1. The aritolonchic acid A linear equation $(\mathrm{y}: \mu \mathrm{V} \bullet \mathrm{s} ; \mathrm{x}: \mathrm{mg} / \mathrm{mL})$ and linear range was as follows: $\mathrm{Y}=4109.3+311855.4 \mathrm{X}(\mathrm{r}=0.999), 0.0094-0.6 \mathrm{mg} / \mathrm{mL}$.

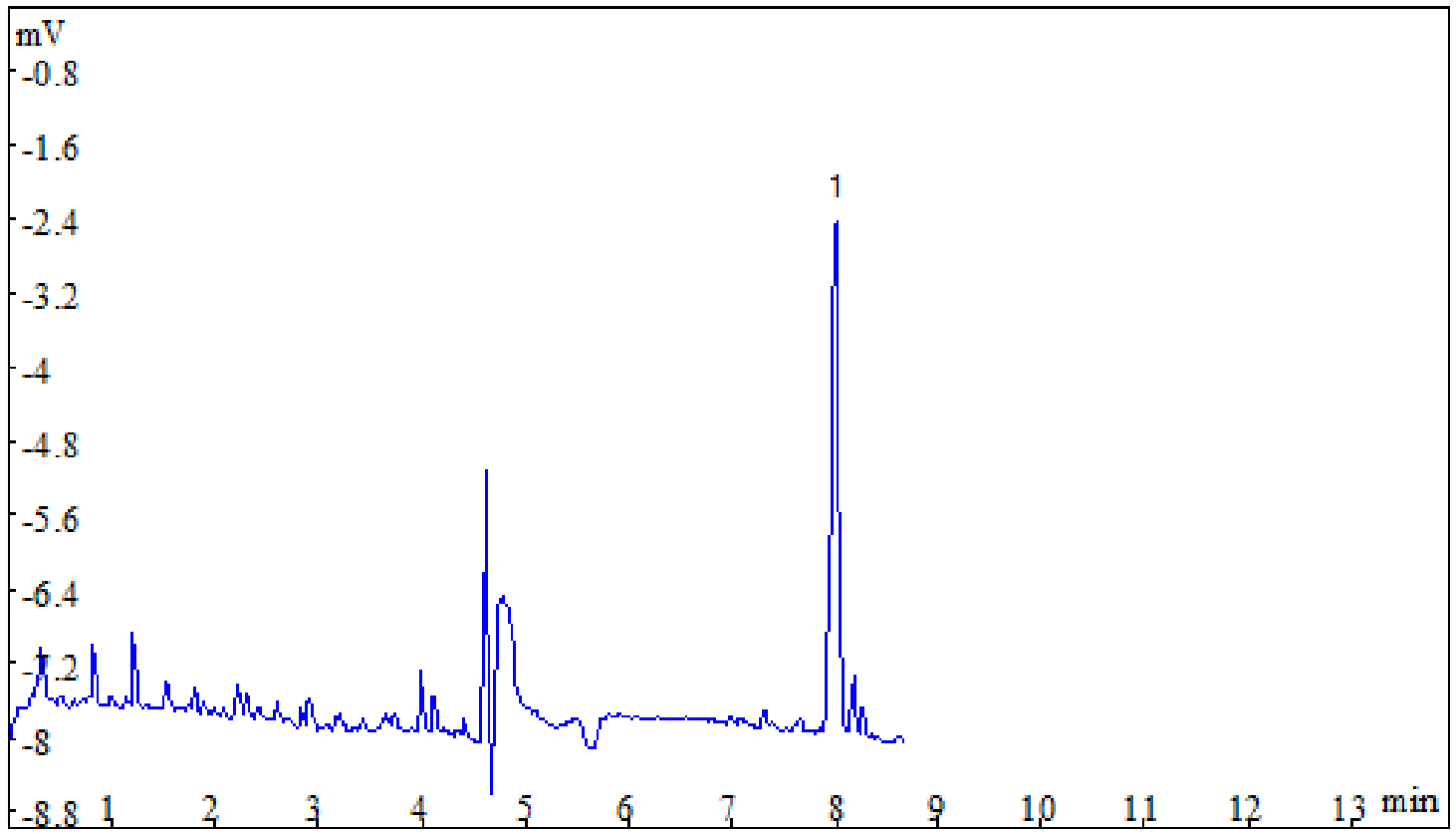

Fig.1 Electrophorogram of aritolonchic acid A standard solution 1-aritolonchic acid A 


\subsection{Precision test.}

The aritolonchic acid A standard solution was continuous injected 5 times. The RSD of migration time and peak area were $5.8 \%$ and $2.6 \%$ for aritolonchic acid A.

\subsubsection{Determination of sample content.}

The Xinqin granules sample solution was operated using the separation conditions. The Xinqin granules sample solution chromatogram was showed in Figure 2. The content of aritolonchic acid A in Xinqin granules was $2.87 \mathrm{mg} / \mathrm{g}(\mathrm{RSD}=2.9 \%)(\mathrm{n}=6)$.

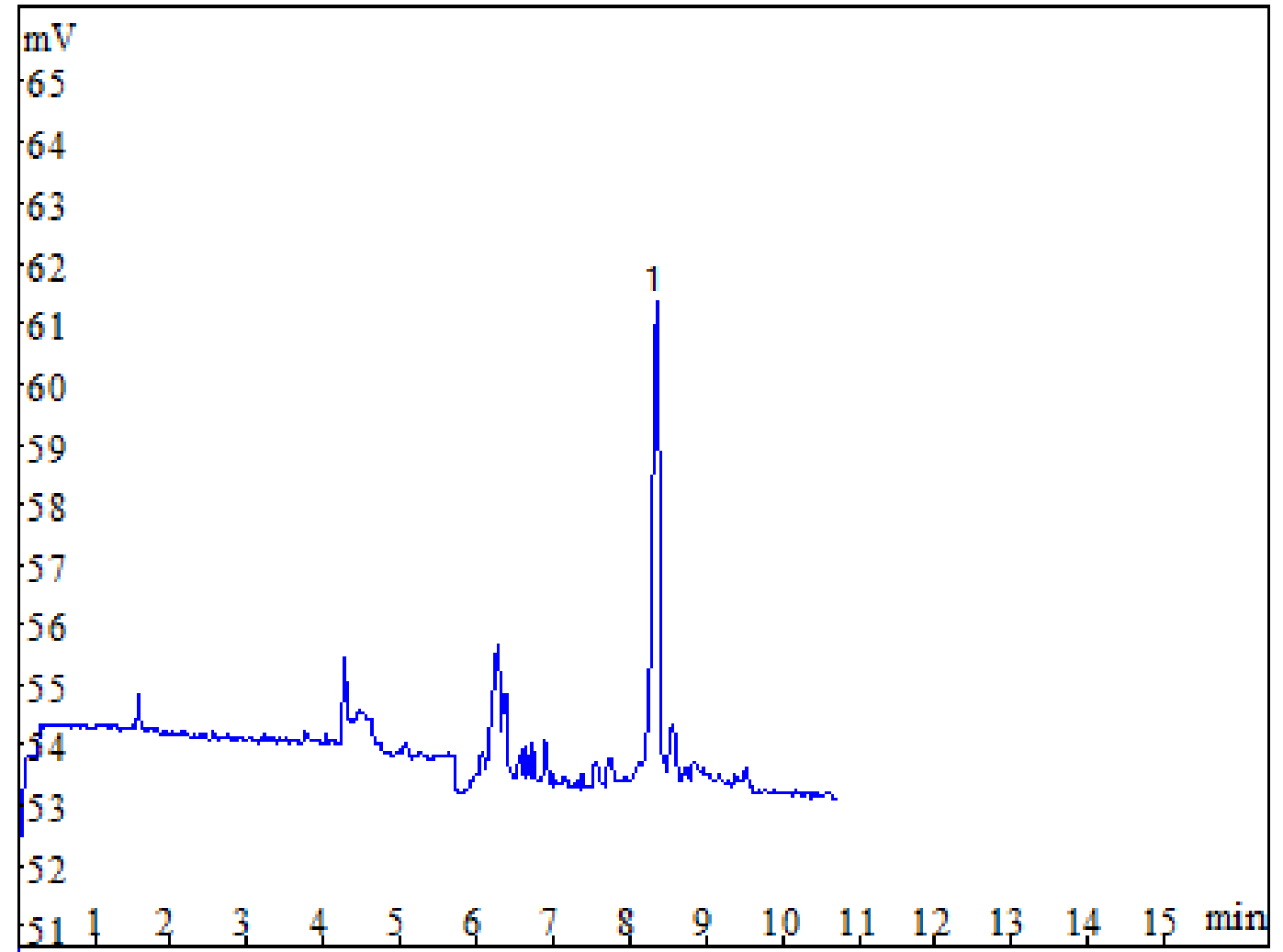

Fig.2 Electrophorogram of Xinqin granules sample solution 1-aritolonchic acid A

\subsubsection{Recovery.}

The recovery of aritolonchic acid A in Xinqin granules sample was in the range of $84.5 \%-126.7 \%(\mathrm{n}=5)$ for five times testing. The average recovery was $105.9 \%(\mathrm{n}=5)$.

\section{Conclusion}

The paper studied limited determination aritolonchic acid A content in Xinqin granules using capillary electrophoresis. The content of aritolonchic acid A in Xinqin granules was $2.87 \mathrm{mg} / \mathrm{g}(\mathrm{RSD}=2.9 \%)(\mathrm{n}=6)$.

\section{Acknowledgments}

Shandong Province Natural Science Foundation: ZR2010BL025.

\section{References}

1. Yan J.S., Li J.H., Sun G.J., Liu F.Y., (2011) Trace Analysis of Aristolochic Acid A in RADIX ET RHIZOMA ASART from Different Production
Areas, Journal of Anhui Agri. Sci., 39 (32), 19745-19746

2. Wang L., Ding L.Y., Ding F., Li C.X., Zhu X.H., Hao L., (2009) Experimental Study on Reducing the Dissolution of Aristolochic Acid A in Aristolochiae manshuriensis by Different Doses of Bamboo Leaf, Lishizhen Medicine and Materia Medica Research, 20(11): 2681-2682

3. Liu M.F., Liu J., Lv H.R., Zhou H., (2011) Study on Optimization of Extract Process of Aristolochic Debilis and Toxicity Reduction by Different Processing, Guangzhou Chemical, 39(9): 81-85

4. Zhang P., (2011) The assay of aristolochic acid A in different organs of Saruma, Northern Horticulture, 20: 177-178

5. Qiu Q., Qin C.H., Huang Q.Y., (2018) Determination of aristolochic acid A in Shennong snake liquor by HPLC, Journal of Guangxi Medical University, 35(1): 116-118

6. Ge X.Z., Zhang Y.P., Cai X.P., Wu C.Q., Wang H.B., (2010) Limit Test for Aristolochic Acid A in Xinqin Granules by HPLC, Chinese Journal of Pharmaceuticals, 41(8): 604-606 
7. Pan Y.K., Gan Q., (2009) Determination of aristolochic acid A in prepared traditional Chinese medicines by LC-MS/MS, Physical and chemical examination, 45: 1017-1023

8. Li W., Han J.P., Gao J., Liu C.X., (2007) Determination of Aristolochic Acid A in Rhizoma Asari and Yangxue Qingnao Granules Using Liquid Chromatography Tandem Mass Spectrometry, Chinese Journal of Analytical Chemistry, 35(12): 1798-1800

9. Zhou W., Liu H.W., Chen L.R., Cheng X.G., Li J., (2005,) HPLC/MS Analysis of Aristolochic Acid A in Chinese Traditional Patent Medicine, Chemical Research, 16(4): 80-82

10. Zhou W., Niu H.L., Wang B., Liu H.W., Jia S.T.,
Xie Y.S., (2011) Research of Aristolochic Acid A in Chinese Traditional Patent Medicine by UPLC/MS, Chinese Journal of Spectroscopy Laboratory, 28(1): 183-187

11. Nan T.G., He S.P., Tan G.Y., Li G., Wang B.M., Huang L.Q., (2010) Development of Sensitive Monoclonal Antibody-based Enzyme-linked Immunosorbent Assay for Renal Toxicity Ingredient Aristolochic acid A in Chinese Herb Medicine, Chinese Journal of Analytical Chemistry, 38(8): 1206-1210

12. Lu Q.H., (2015) Limit test of aristolochic acid A in Zhuifeng Tougu capsule, Chin. J. Mod. Drug Appl., 9(15), 285-286 